\title{
Gravedad de caries empleando un criterio del tamaño de la lesión y variables asociadas en una muestra de escolares mexicanos
}

\section{Caries severity using a criterion according to size of lesion among Mexican schoolchildren}

\author{
Ana Alicia Vallejos-Sánchez ${ }^{1}$, Mirna Minaya-Sánchez ${ }^{1}$, Juan Fernando Casanova-Rosado¹, \\ Alejandro José Casanova-Rosado ${ }^{1}$, Julieta Macias-Ortega ${ }^{2}$, Sergio Vera-Guzmán², Carlo Eduardo Medina-Solís ${ }^{2}$
}

Forma de citar: Vallejos Sánchez AA, Minaya-Sánchez M, Casanova-Rosado JF, Casanova-Rosado AJ, MaciasOrtega J, Vera Guzmán S, et al. Gravedad de caries empleando un criterio del tamaño de la lesión y variables asociadas en una muestra de escolares mexicanos. Rev Univ Ind Santander Salud. 2015; 47(3): 291-299. DOI: http://dx.doi.org/10.18273/revsal.v47n3-2015005 (c) (1) Ð

\section{RESUMEN}

Objetivo: Determinar la gravedad de caries (tamaño de la lesión) y las variables asociadas en niños escolares mexicanos. Materiales y Métodos: Se realizó un estudio transversal en 1373 escolares de 6 a 12 años de edad inscritos en escuelas públicas de Campeche, México. Se distribuyeron cuestionarios para determinar una serie de variables sociodemográficas, socioeconómicas y conductuales. Se realizó un examen bucal a cada niño para establecer el tamaño de las lesiones de caries de acuerdo al índice llamado "criterio de magnitud de la lesión cariosa" y asignarlo de acuerdo al número de lesiones presentes a uno de 4 grupos de riesgo para desarrollar caries, en donde el primer grupo representa a los menos afectados. Se generó un modelo multivariado de regresión logística multinomial. Resultados: Los grupos de riesgo a caries quedaron distribuidos de la siguiente manera: $598(43.6 \%)$ fueron del grupo 1; $261(19.0 \%) \mathrm{se}$ asignaron al grupo $2 ; 316(23.0 \%)$ estuvieron en el grupo 3; y 198 (14.4\%) en el grupo 4. Comparando con el grupo menos afectado por lesiones cariosas, las variables asociadas en los diferentes grupos de gravedad en el modelo multivariado fueron: grupo de riesgo 2: edad, sexo y defectos de desarrollo del esmalte; grupo de riesgo 3: atención dental en el último año, actitud de la madre hacia la salud bucal y defectos de desarrollo del esmalte; grupo de riesgo 4: edad, atención dental en el último año, actitud de la madre hacia la salud bucal y defectos de desarrollo del esmalte. Conclusiones: La gravedad de caries está influenciada por variables demográficas, conductuales y clínicas.

Palabras clave: salud bucal, caries dental, gravedad del paciente, esmalte dental.

1. Universidad Autónoma de Campeche. Campeche, México.

2. Instituto de Ciencias de la Salud de la Universidad Autónoma del Estado de Hidalgo. Pachuca, México.

Correspondencia: Carlo Eduardo Medina. Dirección: Avenida del Álamo \# 204, Fraccionamiento Paseo de los Solares. Colonia Santiago Tlapacoya. CP. 42110. Pachuca de Soto, Hidalgo, México. Correo electrónico: cemedinas@yahoo.com. Teléfono: 01 (771) 1483338. 


\section{ABSTRACT}

Objective: To determine the severity of caries (lesion size) and associated variables in Mexican schoolchildren. Material and Methods: A cross-sectional study was conducted in 1373 schoolchildren 6-12 years old enrolled in public schools in Campeche, Mexico. Questionnaires were distributed to determine demographic, socioeconomic and behavioral variables. Dental examination was performed to each child to establish the size of caries lesions according "Criterion of carious lesion magnitude" index and to allocate according to the number of lesions present to one of four risk groups for developing cavities wherein the first group represents the least affected. A multivariate multinomial logistic regression model was generated. Results: Caries risk groups were distributed as follows: 598 $(43.6 \%)$ were in group 1; $261(19.0 \%)$ were assigned to group 2; $316(23.0 \%)$ were in group 3; and $198(14.4 \%)$ in group 4. Comparing with the least affected group by carious lesions, associated variables for severity in the multivariate model were "Risk Group 2": age, sex and developmental enamel defects; "Risk Group 3": dental care in the past year, mother's attitude toward oral health and developmental enamel defects; "Risk Group 4": age, dental care in the past year, mother's attitude toward oral health and developmental enamel defects. Conclusions: Severity of decay is influenced by demographic, behavioral and clinical variables.

Keywords: oral health, dental caries, patient acuity, dental enamel.

\section{INTRODUCCIÓN}

La Organización Mundial de la Salud (OMS) ha definido la caries dental como un proceso localizado de origen multifactorial que se inicia después de la erupción dentaria, determinando el reblandecimiento de los tejidos duros del diente, evolucionando hasta la formación de una cavidad ${ }^{1}$. Diversos estudios realizados alrededor del mundo mencionan que entre el 60 a $90 \%$ de los niños escolares se encuentran afectados por caries $^{2}$. Además, es la necesidad de salud más frecuentemente no cubierta en preescolares, escolares y adolescentes de Latinoamérica, sobre quienes se observa poca experiencia de tratamiento dental restaurador ${ }^{3-6}$. Específicamente en México, la caries dental representa el principal problema de salud pública bucal; por ejemplo, en la dentición permanente entre el 70 y $85 \%$ de la población escolar a los 12 años de edad presenta esta enfermedad ${ }^{7-10}$. Constituye, por otro lado, la primera causa de mortalidad dental en varios grupos de edad ${ }^{11}$ representando un importante desafío para el sistema de atención a la salud bucal, debido a los altos costos de atención.

Uno de los retos para la salud pública bucal es el conocimiento actualizado de las cifras sobre la prevalencia e incidencia de las enfermedades y alteraciones bucodentales en las poblaciones. El contar con información actualizada es un aspecto esencial para poder mejorar la salud de la población a través de políticas y programas de salud pertinentes, así como para realizar evaluaciones del impacto de éstas cuando han sido instauradas ${ }^{12,13}$. Existen diversas formas para determinar el estado de caries que presenta la población, para expresar la prevalencia y la intensidad o gravedad de la caries dental, los índices mundialmente utilizados 292 son el CEOD (Promedio de Dientes Cariados, Extraídos/ Perdidos y Obturados) y el CPOD (Promedio de Dientes Permanentes Cariados, Perdidos y Obturados) que se refieren al promedio de dientes cariados, perdidos y obturados para la dentición primaria y permanente, respectivamente ${ }^{14}$. Sin embargo, diversos autores han cuestionado su certeza en la evaluación de caries por diversas razones ${ }^{15,16}$. Varios estudios han sido realizados para determinar la cantidad de caries que se presenta en la población. En ellos se realizan diversos puntos de corte para separar a los sujetos de bajo y alto nivel de caries o utilizando el índice de caries significante $(\mathrm{SiC})^{17-19}$ sin embargo, no toman en cuenta la gravedad de dichas lesiones.

La evidencia epidemiológica sugiere la existencia de variables asociadas a la caries dental en la dentición permanente, entre ellas se encuentran: edad, sexo, etnicidad, actitud de la madre, presencia de defectos de desarrollo del esmalte, placa dentobacteriana, atención dental, presencia de dolor, así como diversos indicadores de posición socioeconómica; como ingreso, tipo de escuela, hacinamiento y área geográfica ${ }^{6,20-27}$. Estudios epidemiológicos sobre prevalencia de caries dental han sido realizados en México, pero tampoco toman en cuenta el tamaño de la lesión. El objetivo del presente trabajo fue determinar la gravedad de caries (tamaño de la lesión) y las variables asociadas en niños escolares mexicanos.

\section{MATERIAL Y MÉTODOS}

Diseño, población y muestra del estudio: Este trabajo es un análisis secundario de un estudio transversal sobre salud bucal en escolares de 6 a 12 años de edad asistentes 
a escuelas urbanas primarias de la ciudad de Campeche, Campeche, México. Parte de la metodología ha sido publicada previamente, ${ }^{48-32}$. De manera breve, en el Estado está permitida la distribución de sal fluorurada ya que el agua potable que se distribuye en la red pública no contiene fluoruro de forma natural. Se seleccionaron por muestreo aleatorio simple cuatro escuelas primarias públicas que se encuentran bajo el programa de salud bucal del Instituto Mexicano del Seguro Social (IMSS), con una población total de 1603 sujetos. Previo acuerdo con directores y profesores, se distribuyeron cartas de consentimiento informado entre los padres de familia en las cuales se explicaba el objetivo del estudio y se invitaba a participar a sus hijos. Se obtuvo una tasa de respuesta de $87.5 \%(n=1403)$. Después de aplicados los criterios de inclusión y exclusión (niños mayores de
6 y menores de 12, que estuvieran inscritos en alguna escuela seleccionada y que la madre/tutor hubiera firmado la carta de consentimiento informado) se reunió una muestra final de 1373 niños $(85.7 \%$ de la población total original).

\section{Variables del estudio}

La variable dependiente fue la gravedad de caries. Para la detección de caries se aplicó el Criterio de Magnitud de la Lesión Cariosa (CMLC) propuesto por Gutiérrez y Morales $^{33}$ y es descrito en la Tabla 1A. Este criterio asigna al sujeto a uno de cuatro grupos de gravedad de acuerdo al número y magnitud de las lesiones cariosas clínicamente observables en cada diente. Los grupos de gravedad se muestran en la Tabla 1B.

Tabla 1A. Criterio de Magnitud de la Lesión Cariosa (CMLC) propuesto por Gutiérrez \& Morales (1987).

\begin{tabular}{ll}
\hline Código & Magnitud de la lesión \\
\hline 0 & Si el diente está libre de caries clínicamente observable. \\
Lesión grado 1 & Si el diente presenta pigmentación oscura en fosas y fisuras sin presencia de tejido reblandecido. \\
Lesión grado 2 & Si el diente presenta caries limitada a surcos y fisuras con presencia de tejido reblandecido. \\
Lesión grado 3 & $\begin{array}{l}\text { Si el diente presenta pérdida evidente de tejido coronal, debida a caries, menor a dos terceras partes de } \\
\text { la corona clínica. }\end{array}$ \\
Lesión grado 4 & $\begin{array}{l}\text { Si el diente presenta pérdida evidente de tejido coronal, debida a caries, mayor a dos terceras partes de } \\
\text { la corona clínica o presencia de restos radiculares. }\end{array}$ \\
$\overline{2}$ & Si el diente presenta una obturación clase I con material definitivo y en buen estado. \\
$\overline{3}$ & Si el diente presenta una obturación clase II con material definitivo y en buen estado. \\
$\overline{4}$ & $\begin{array}{l}\text { Si el diente presenta una obturación de } 3 \text { o más superficies con material definitivo y en buen estado o } \\
\text { bien una corona. }\end{array}$ \\
Los dientes ausentes & se clasifican con $\mathbf{N}$, los extraídos por caries con E y los ausentes congénitamente o extraídos por \\
traumatismo con una a.
\end{tabular}

Tabla 1B. Los cuatro grupos de gravedad derivados de la administración del Criterio de Magnitud de la Lesión Cariosa (CMLC).

\begin{tabular}{ll}
\hline Gravedad & Número y grado de caries \\
\hline Grupo 1 & Sujetos libres de caries o sujetos con 1 a 4 lesiones grado 1. \\
Grupo 2 & Sujetos con más de 4 lesiones grado 1 o sujetos con 1 a 3 lesiones grado 2. \\
Grupo 3 & Sujetos con más de 3 lesiones grado 2 o sujetos con 1 a 3 lesiones grado 3. \\
Grupo 4 & Sujetos con más de 3 lesiones grado 3 o sujetos con 1 o más lesiones grado 4. \\
\hline
\end{tabular}

Las variables independientes fueron edad, sexo, frecuencia de cepillado, inicio de uso de pasta dental, número de fuentes de flúor, caries en la dentición primaria, escolaridad de la madre, actitud de la madre hacia la salud bucal de su hijo (utilizando los instrumentos descritos en otros estudios) $)^{3,4,6,13,28}$, presencia de fluorosis (índice de Dean), y presencia de defectos de desarrollo del esmalte (índice de defectos de desarrollo del esmalte), específicamente opacidades del esmalte, clasificadas como demarcadas o difusas, al igual que hipoplasias del esmalte ${ }^{34}$. 
Recolección de los datos: Para la recolección de los datos se utilizó un cuestionario estructurado dirigido a las madres, el cual fue entregado y recolectado a través de las escuelas, para determinar las variables sociodemográficas (edad, sexo), socioeconómicas (escolaridad de la madre), conductuales (frecuencia de cepillado), y de exposición a fluoruros durante los primeros 6 años de vida (número de fuentes adicionales de fluoruro e inicio de uso de pasta). Con relación al número de fuentes adicionales de fluoruros se estableció si el niño había estado expuesto a fluoruros en los primeros seis años de vida (no importando las veces al año), en términos de gotas, enjuagues (consultorios, casa o escuela), aplicaciones por parte del dentista, auto aplicaciones de fluoruro en gel y algún suplemento con fluoruro.

Todos los sujetos fueron examinados clínicamente en un sillón dental fijo por uno de tres dentistas capacitados y estandarizados en el criterio para la detección de caries (kappa $=0,92)$ y fluorosis dental (kappa $=0,85$ ). Para la medición de las variables clínicas se usó un espejo plano y luz natural, se secó el diente, si era necesario, con gasa estéril.

Análisis estadístico: Todos los análisis se hicieron empleando el paquete estadístico Stata 9.0. Se calcularon para las variables continuas medidas de tendencia central y de dispersión. En el caso de las variables categóricas, se obtuvieron las frecuencias para cada categoría, así como el porcentaje correspondiente. En el análisis bivariado se emplearon las pruebas de Chi cuadrado y Kruskall-Wallis de acuerdo a la escala de medición de las variables a contrastar. Finalmente, conformamos un modelo multivariado de regresión logística multinomial con el propósito de obtener las mejores variables asociadas con la presencia de caries dental en la dentición permanente. La fuerza de la asociación en nuestro modelo es expresado como razones de momios con sus intervalos de confianza al 95\% (IC 95\%), reportándose igualmente los valores de $\mathrm{p}$.

\section{RESULTADOS}

La muestra incluyó 1373 escolares de 6 a 12 años de edad. Los resultados del análisis descriptivo se muestran en la Tabla 2; el promedio de edad fue de $8.84 \pm 1.80$ años. Las mujeres representaron el $51.3 \%$. La escolaridad de la madre fue de $8.91 \pm 4.12$ años.
La distribución de los grupos de riesgo para caries quedó de la siguiente manera: 598 (43.6\%) fueron de grupo $1 ; 261(19.0 \%)$ se asignaron al grupo $2 ; 316$ (23.0\%) estuvieron en el grupo 3; y 198 (14.4\%) en el grupo 4.

Tabla 2. Características de los escolares incluidos en el estudio.

\begin{tabular}{|c|c|c|}
\hline Variable & Media \pm DE & Límites \\
\hline Edad & $8.84 \pm 1.80$ & $6-12$ \\
\hline \multirow[t]{2}{*}{ Escolaridad de la madre } & $8.91 \pm 4.12$ & $0-20$ \\
\hline & $\mathbf{n}$ & Porcentaje \\
\hline \multicolumn{3}{|l|}{ Sexo } \\
\hline Hombres & 669 & 48.7 \\
\hline Mujeres & 704 & 51.3 \\
\hline \multicolumn{3}{|l|}{ Frecuencia de cepillado } \\
\hline Menos de 7 veces/semana & 133 & 9.7 \\
\hline Al menos una vez al día & 1240 & 90.3 \\
\hline \multicolumn{3}{|l|}{ Inicio de uso de pasta dental } \\
\hline Después de los 4 años & 968 & 70.5 \\
\hline Antes de los 4 años & 405 & 29.5 \\
\hline \multicolumn{3}{|l|}{ Atención dental (último año) } \\
\hline No & 473 & 34.5 \\
\hline $\mathrm{Si}$ & 900 & 65.5 \\
\hline \multicolumn{3}{|l|}{ Actitud de la madre } \\
\hline Negativa & 373 & 27.2 \\
\hline Positiva & 1000 & 72.8 \\
\hline \multicolumn{3}{|l|}{ Defectos del esmalte } \\
\hline Sin defecto & 1270 & 92.5 \\
\hline Con defecto & 103 & 7.5 \\
\hline \multicolumn{3}{|l|}{ Fluorosis } \\
\hline Sin fluorosis & 661 & 48.2 \\
\hline Muy leve & 603 & 43.9 \\
\hline Leve, moderada, severa & 109 & 7.9 \\
\hline
\end{tabular}

En la Tabla 3 se pueden observar los resultados del análisis bivariado. Se observó mayor promedio de edad en los escolares del grupo de riesgo 2 y menor en los del grupo 4 (9.29 vs $8.31 ; \mathrm{p}<0.001)$. Además también observamos diferencia estadísticamente significativa en cuanto al sexo $(\mathrm{p}<0.05)$, la atención dental en el último año $(\mathrm{p}<0.05)$, la actitud de la madre hacia la salud bucal $(\mathrm{p}<0.001)$, los defectos en el esmalte $(\mathrm{p}<0.001)$, y la fluorosis dental. No se observaron diferencias estadísticamente significativas de la gravedad de caries por escolaridad de la madre, frecuencia de cepillado dental y edad de inicio del uso de pasta. 
Gravedad de caries empleando un criterio del tamaño de la lesión y variables asociadas en una muestra de escolares mexicanos

Tabla 3. Análisis bivariado entre la gravedad de caries dental y las variables independientes.

\begin{tabular}{|c|c|c|c|c|c|}
\hline \multirow{2}{*}{ Variable } & Grupo 1 & Grupo 2 & Grupo 3 & Grupo 4 & Valor de $p$ \\
\hline & $598(43.6)$ & $261(19.0)$ & $316(23.0)$ & $198(14.4)$ & \\
\hline Edad & $8.79 \pm 1.80$ & $9.29 \pm 1.79$ & $8.85 \pm 1.83$ & $8.31 \pm 1.59$ & $<0.001 *$ \\
\hline Escolaridad de la madre & $9.13 \pm 3.96$ & $8.96 \pm 4.61$ & $8.70 \pm 3.94$ & $8.53 \pm 4.15$ & $0.2132 *$ \\
\hline \multicolumn{6}{|l|}{ Sexo } \\
\hline Hombres & $301(45.0)$ & $106(15.8)$ & $160(23.9)$ & $102(15.3)$ & \\
\hline Mujeres & $297(42.2)$ & $155(22.0)$ & $156(22.2)$ & $96(13.6)$ & $0.036 \dagger$ \\
\hline \multicolumn{6}{|l|}{ Frecuencia de cepillado } \\
\hline Menos de 7 veces/semana & $58(43.6)$ & $20(15.0)$ & $32(24.1)$ & $23(17.3)$ & \\
\hline Al menos una vez al día & $540(43.6)$ & $241(19.4)$ & $284(22.9)$ & $175(14.1)$ & $0.545 \dagger$ \\
\hline \multicolumn{6}{|l|}{ Inicio de uso de pasta dental } \\
\hline Después de los 4 años & $407(42.1)$ & $188(19.4)$ & $236(24.4)$ & $137(14.1)$ & \\
\hline Antes de los 4 años & $191(47.2)$ & $73(18.0)$ & $80(19.7)$ & $61(15.1)$ & $0.185 \dagger$ \\
\hline \multicolumn{6}{|l|}{ Atención dental (último año) } \\
\hline No & $223(47.2)$ & $99(20.9)$ & $99(20.9)$ & $52(11.0)$ & \\
\hline $\mathrm{Si}$ & $375(41.7)$ & $162(18.0)$ & $217(24.1)$ & $146(16.2)$ & $0.013 \dagger$ \\
\hline \multicolumn{6}{|l|}{ Actitud de la madre } \\
\hline Negativa & $104(27.9)$ & $62(16.6)$ & $112(30.0)$ & $95(25.5)$ & \\
\hline Positiva & $494(49.4)$ & $199(19.9)$ & $204(20.4)$ & $103(10.3)$ & $<0.001 \uparrow$ \\
\hline \multicolumn{6}{|l|}{ Defectos del esmalte } \\
\hline Sin defecto & $574(45.2)$ & $242(19.1)$ & $277(21.8)$ & $177(13.9)$ & \\
\hline Con defecto & $24(23.3)$ & $19(18.4)$ & $39(37.9)$ & $21(20.4)$ & $<0.001 \uparrow$ \\
\hline \multicolumn{6}{|l|}{ Fluorosis } \\
\hline Sin fluorosis & $261(39.5)$ & $134(20.3)$ & $152(23.0)$ & $114(17.2)$ & \\
\hline Muy leve & $283(46.9)$ & $108(17.9)$ & $143(23.7)$ & $69(11.5)$ & \\
\hline Leve, moderada, severa & $54(49.5)$ & $19(17.4)$ & $21(19.3)$ & $15(13.8)$ & $0.027 \dagger$ \\
\hline
\end{tabular}

* Kruskal-Wallis test, $\uparrow$ Chi cuadrado

El análisis multivariado de regresión logística multinomial se presenta en la tabla 4. Para el grupo de riesgo a caries 2 (sujetos con más de 4 lesiones grado 1 o sujetos con 1 a 3 lesiones grado 2), las variables asociadas fueron edad, sexo y defectos estructurales del esmalte: observamos que conforme aumenta la edad la posibilidad de presentar lesiones de caries aumentaron 16\% (IC95\%= 7 - 26). Además, las mujeres tuvieron mayor posibilidad ( $\mathrm{RM}=1.51$; IC95\% $=1.12-2.03$ ) de tener lesiones cariosas que los varones; y los sujetos con defectos de desarrollo del esmalte fueron $1.90($ IC95\%= $1.01-3.55)$ veces más probables de presentar lesiones cariosas que los que no tenían defectos del esmalte. Para el grupo de riesgo a caries 3 (sujetos con más de 3 lesiones grado 2 o sujetos con 1 a 3 lesiones grado 3 ), las variables asociadas fueron: atención dental en el último año, actitud de la madre hacia la salud bucal de su hijo y defectos de desarrollo del esmalte: los escolares que recibieron atención dental en el último año $(\mathrm{RM}=1.36$; $\mathrm{IC} 95 \%=1.01$ - 1.84) y los que tuvieron defectos del esmalte $(\mathrm{RM}=3.07$; IC95\%=
1.79 - 5.25) presentaron mayor posibilidad de tener lesiones cariosas más graves; mientras que los hijos de madres con una actitud positiva hacia la salud bucal presentaron menos $(\mathrm{RM}=0.38$; IC95\% $=0.27$ $0.52)$ posibilidad de tener lesiones de caries graves.

Finalmente, para el grupo de riesgo 4 (sujetos con más de 3 lesiones grado 3 o sujetos con 1 o más lesiones grado 4), resultaron asociadas la edad, la atención dental en el último año, la actitud de la madre hacia la salud bucal de su hijo y los defectos de desarrollo del esmalte: en este grupo, por cada año de edad la probabilidad de tener lesiones graves disminuyó $(\mathrm{p}<0.05)$; los niños con atención dental en el último año tuvieron más posibilidad de presentar lesiones graves $(\mathrm{RM}=2.07$; IC95\%= $1.42-3.02)$, al igual que los niños con defectos de desarrollo del esmalte ( $\mathrm{RM}=2.34$; IC95\%=1.24 - 4.43); mientras que los escolares con una madre con actitud positiva hacia la salud bucal tuvieron menos posibilidad de tener lesiones de caries graves $(\mathrm{RM}=0.19$; IC $95 \%=$ $0.13-0.28)$. 
Tabla 4. Análisis multivariado entre la gravedad de caries dental y las variables independientes incluidas en el estudio.

\begin{tabular}{|c|c|c|c|}
\hline Variable & Grupo 2 & Grupo 3 & Grupo 4 \\
\hline Edad & $1.16(1.07-1.26) \ddagger$ & $0.98(0.90-1.06) \mathrm{n} / \mathrm{s}$ & $0.79(0.72-0.87) \ddagger$ \\
\hline \multicolumn{4}{|l|}{ Sexo } \\
\hline Hombres & $1 *$ & $1^{*}$ & $1^{*}$ \\
\hline Mujeres & $1.51(1.12-2.03) \dagger$ & $0.97(0.73-1.28) \mathrm{n} / \mathrm{s}$ & $0.86(0.62-1.21) \mathrm{n} / \mathrm{s}$ \\
\hline \multicolumn{4}{|c|}{ Atención dental (último año) } \\
\hline No & $1 *$ & $1 *$ & $1 *$ \\
\hline $\mathrm{Si}$ & $0.89(0.65-1.22) \mathrm{n} / \mathrm{s}$ & $1.36(1.01-1.84)^{*}$ & $2.07(1.42-3.02) \ddagger$ \\
\hline \multicolumn{4}{|c|}{ Actitud de la madre } \\
\hline Negativa & $1 *$ & $1 *$ & $1 *$ \\
\hline Positiva & $0.74(0.52-1.07) \mathrm{n} / \mathrm{s}$ & $0.38(0.27-0.52) \ddagger$ & $0.19(0.13-0.28) \ddagger$ \\
\hline \multicolumn{4}{|c|}{ Defectos del esmalte } \\
\hline Sin defecto & $1 *$ & $1 *$ & $1^{*}$ \\
\hline Con defecto & $1.90(1.01-3.55)^{*}$ & $3.07(1.79-5.25) \dagger$ & $2.34(1.24-4.43) \dagger$ \\
\hline
\end{tabular}

$* \mathrm{p}<0.05, \dagger \mathrm{p}<0.01, \$ \mathrm{p}<0.001$

$\mathrm{n} / \mathrm{s}=$ no significativo

En la Tabla 5 se presenta un análisis entre los diferentes grupos de gravedad de caries (criterio de magnitud de la lesión cariosa) y la experiencia de caries empleando los índices ceod y CPOD; se obtuvo diferencia de la experiencia de caries a través de los diferentes grupos de gravedad de caries $(\mathrm{p}<0.05)$.

Tabla 5. Análisis entre los grupos de gravedad de caries y los índices CEOD, CPOD y CEOD+CPOD.

\begin{tabular}{lccccc}
\hline & Grupo 1 & Grupo 2 & Grupo 3 & Grupo 4 & Valor p \\
\hline CEOD (n=763) & $0.01 \pm 0.10$ & $1.53 \pm 1.10$ & $3.75 \pm 1.87$ & $6.22 \pm 2.27$ & $\begin{array}{c}0.0001 * \\
<0.001 \dagger\end{array}$ \\
\hline CPOD (n=1373) & $0.008 \pm 0.107$ & $1.70 \pm 1.49$ & $1.77 \pm 1.97$ & $1.16 \pm 1.71$ & $\begin{array}{c}0.0001 * \\
<0.001 \dagger\end{array}$ \\
\hline CEOD+CPOD (n=1373) & $0.01 \pm 0.14$ & $2.44 \pm 1.45$ & $3.98 \pm 2.22$ & $6.03 \pm 2.92$ & $\begin{array}{c}0.0001 * \\
<0.001 \dagger\end{array}$ \\
\hline
\end{tabular}

*Kruskall-Wallis test,

$\dagger$ Prueba de tendencia no paramétrica.

\section{DISCUSIÓN}

Este estudio empleó un sistema que evalúa el estado de la caries dental en escolares tomando en cuenta el tamaño de la lesión. Se encontró que el mayor porcentaje de niños se ubicó en el grupo 1, esto es los que están menos gravemente afectados (sujetos libres de caries o sujetos con 1 a 4 lesiones grado 1). También, los hallazgos demuestran asociaciones entre variables sociodemográficas, socioeconómicas y conductuales asociadas a la gravedad de caries. Pocos han sido los estudios que han empleado este criterio para el estudio de la gravedad de caries dental, por lo que las comparaciones tendrán esta limitación.

Uno de los indicadores de riesgo para caries dental que más han sido estudiados son la edad y el sexo. Con relación a la edad, estudios realizados en varias partes del mundo han encontrado consistentemente 296 que cuando la edad se incrementa la presencia de caries aumenta ${ }^{6,20,26,27}$; lógicamente, al tener mayor tiempo de exposición al ambiente ácido dentro de la boca se incrementa la posibilidad de tener lesiones de mayor gravedad. Se observó resultados ligeramente contradictorios, ya que para el grupo 2 si se comportó de esta forma, sin embargo, para el grupo 4 no resultó ser así. Por otro lado, en cuanto al sexo, las mujeres han sido reportadas estar más afectadas que los hombres ${ }^{6,20,24}$. Una de las explicaciones propuestas para esto es la diferencia en el tiempo de erupción de los dientes permanentes, el cual es más temprano en las mujeres que en lo varones, lo que hace que sus dientes están más expuestos al ambiente cariogénico de la boca o el fácil acceso a los alimentos durante la preparación de estos. En este sentido, Lukacs y Largaespada ${ }^{35}$, y Lukacs, ${ }^{36,37}$ han discutido ampliamente esta relación, proponiendo una teoría basada en explicaciones biológicas así como culturales. 
Estudios realizados en México ${ }^{38}$, Venezuela ${ }^{39}$, Brasil $^{25}$, y Nicaragua ${ }^{6}$, son coincidentes con los resultados del presente trabajo. La actitud positiva hacia la salud bucal del niño, por parte de la madre, tiene una influencia sobre diversos aspectos de la salud bucal ${ }^{25,39}$ y la gravedad de las lesiones cariosas ${ }^{6,38}$. Generalmente, los sujetos con mejores actitudes son aquellas con mejor educación y mejor nivel socioeconómico, por lo que las personas que tienen actitud positiva hacia la salud han tenido mejor acceso a información en salud, que les sirve para la formación de hábitos de higiene bucal apropiados así como tener chequeos de rutina con el dentista. Por lo tanto, se puede afirmar que todos estos factores pueden tener una influencia en el cuidado de la condición de la salud oral de los niños ${ }^{40}$.

Es indiscutible que una buena salud bucal puede mejorarse a través de los chequeos dentales regulares, incluso es lo recomendado por diversas agencias de salud y asociaciones de odontología ${ }^{41,42}$. Estas visitas al dentista son importantes ya que durante ellas, las enfermedades bucales se pueden diagnosticar, tratarse, e incluso prevenirse a tiempo, y las pautas de higiene oral personales pueden ser constantes. Sin embargo, algunos estudios reportan porcentajes muy bajos de visitas al dentista en México ${ }^{43}$ o en Nicaragua ${ }^{44}$, donde informan que sólo el 3\% de los niños de 6 a 12 años de edad visitaron a un profesional dental para un chequeo de rutina. Algunos estudios han puesto de manifiesto que las familias de bajo estatus socioeconómico visitan al dentista con más frecuencia debido al dolor o malestar ${ }^{45,46}$. De alguna forma, esto es confirmado por el hallazgo de nuestro estudio, al encontrar una asociación entre la severidad de caries y la atención dental; reflejando que los escolares están utilizando los servicios de salud bucal quizá por motivos curativos. Estudios alrededor del mundo han encontrado que la presencia de defectos de desarrollo del esmalte está asociada a la presencia de caries dental en dentición permanente. En Brasil, Jeremias et al. ${ }^{21}$, encontraron una estrecha relación entre estas variables en niños de 6 a 12 años de edad. En un estudio realizado en Eslovenia observaron que los niños de 6 a 12 años que presentaron defectos del esmalte tuvieron significativamente mayor experiencia de caries en su dentición permanente ${ }^{22}$. Los defectos del esmalte permiten que la placa dentobacteriana se adhiera más que en el esmalte normal, por lo que los microorganismos causantes de la caries pueden permanecer mayor tiempo y provocar desmineralización.

Este trabajo tiene ciertas limitaciones que deben ser tenidas en cuenta para la interpretación de los resultados. El diseño transversal, es una de ellas, debido a que se mide al mismo tiempo la causa y el efecto, por lo que no es posible establecer relaciones causales sino sólo asociaciones estadísticas. Por otro lado, pocos estudios han utilizado el criterio para caries utilizado en el presente estudio, por lo que las comparaciones tuvieron esta limitación.

\section{CONCLUSIONES}

Los hallazgos del presente estudio permiten concluir que la gravedad de caries está asociada con diferentes tipos de variables, incluyendo indicadores demográficos como la edad y el sexo; de tipo conductual como la atención dental en el último año y la actitud de la madre hacia la salud bucal; al igual que factores clínicos como los defectos estructurales del esmalte. Es necesario destacar que la utilización de indicadores de caries que evalúen el tamaño de la lesión, más que sólo la presencia o ausencia de caries, deben ser estudiados con mayor profundidad para determinar la epidemiología de la caries dental. Esto además de aportar información sobre el estado de salud bucal, con el tamaño de la lesión se puede determinar también las necesidades de tratamiento que son más serias y que necesitan ser atendidas con mayor prontitud.

\section{CONSIDERACIONES ÉTICAS}

Este estudio cumple con las especificaciones de protección de los participantes en el estudio y se adhiere a las normas éticas de la Facultad de Odontología de la Universidad Autónoma de Campeche. Los participantes fueron informados sobre los objetivos del estudio, su derecho a participar o no, los resultados y los beneficios. Los datos fueron analizados de forma anónima. Se obtuvieron las cartas de consentimiento informado, y los participantes fueron informados de su estado de salud oral.

\section{CONFLICTOS DE INTERÉS}

Declaramos que no existe conflicto de interés.

\section{REFERENCIAS}

1. World Health Organization. Oral Health Survey - Basics Methods. 4 ed. Geneva: World Health Organization; 1997.

2. Petersen PE, Bourgeois D, Ogawa H, EstupinanDay S, Ndiaye C. The global burden of oral diseases and risks to oral health. Bull World Health Organ. 2005; 83: 66-69. 
3. Herrera MS, Medina-Solís CE, Minaya-Sánchez M, Pontigo-Loyola AP, Villalobos-Rodelo JJ, IslasGranillo H, et al. Dental plaque, preventive care, and tooth brushing associated with dental caries in primary teeth in schoolchildren of Leon, Nicaragua ages 6-9 years. Med Sci Monit. 2013; 19: 10191026.

4. Medina-Solís CE, Vallejos-Sánchez AA, MinayaSánchez M, Casanova-Rosado JF, De la RosaSantillana R, Escoffié-Ramirez M, et al. Cuidados de la dentición primaria de escolares de 6 y 7 años de edad. SIIC Salud. 2013; 20: 128-133.

5. García-Cortés JO, Mejia-Cruz JA, Medina-Cerda E, Orozco-De la Torre G, Medina-Solís CE, MárquezRodriguez $\mathrm{S}$, et al. Experiencia, prevalencia, severidad, necesidades de tratamiento para caries dental e índice de cuidados en adolescentes y adultos jóvenes mexicanos. Rev Invest Clin. 2014; 66: 505-511.

6. Herrera MS, Medina-Solís CE, Islas-Granillo $\mathrm{H}$, Lara-Carrillo E, Scougall-Vilchis RJ, Escoffié-Ramírez M, et al. Sociodemographic, socioeconomic, clinical and behavioral factors modifying experience and prevalence of dental caries in the permanent dentition. West Indian Med J. 2015; 19: 1019-1026

7. Irigoyen ME, Mejía-González A, Zepeda-Zepeda MA, Betancourt-Linares A, Lezana-Fernández MÁ, Álvarez-Lucas CH. Dental caries in Mexican schoolchildren: a comparison of 1988-1989 and 1998-2001 surveys. Med Oral Patol Oral Cir Bucal. 2012; 17: e825-832.

8. Villalobos-Rodelo JJ, Medina-Solís CE, VerdugoBarraza L, Islas-Granillo $\mathrm{H}$, García-Jau RA, Escoffié-Ramírez M, et al. Experiencia de lesiones cariosas reversibles y no-reversibles en escolares mexicanos de 11 y 12 años de edad: un análisis de regresión binomial negativa. Biomedica. 2013; 33: 88-98.

9. Maupome G, Martínez-Mier EA, Holt A, MedinaSolís CE, Mantilla-Rodríguez A, Carlton B. The association between geographical factors and dental caries in a rural area in Mexico. Cad Saude Publica. 2013; 29: 1407-1414.

10. Medina-Solis CE, Maupomé G, Pérez-Núñez R, Avila-Burgos L, Pelcastre-Villafuerte B, PontigoLoyola AP. Política de salud bucal en México: Disminuir las principales enfermedades de salud bucal. Rev Biomed. 2006; 17: 269-286.

11. Medina-Solís CE, Pontigo-Loyola AP, PérezCampos E, Hernández-Cruz P, De la Rosa-Santillana R, Navarrete-Hernandez JJ, et al. Principales razones de extracción de dientes permanentes en una muestra de adultos mexicanos. Rev Invest Clin. 2013; 65: 141-149.

12. Sales-Peres SHC, Bastos JRM. An epidemiological profile of dental caries in 12-year-old children residing in cities with and without fluoridated water supply in the central western area of the State of São Paulo, Brazil. Cad Saude Publica. 2002; 18(5): 1281-1288.

13. Secretaría de Salud. Encuesta Nacional de caries dental 2001. Secretaría de Salud; México DF: 2006.

14. Sheiham A, Maizels J, Maizels A. New composite indicators of dental health. Community Dent Health. 1987; 4(4): 407-414.

15. Segovia-Villanueva A, Estrella-Rodríguez R, Medina-Solís CE, Maupomé G. Severidad de caries en preescolares bajo un programa de odontología preventiva. Rev Salud Publica. 2005; 7: 56-69.

16. Baginska J, Rodakowska E, Kierklo A. Status of occlusal surfaces of first permanent molars in 6-8-year-old children evaluated by the CAST and DMF indices. Eur J Paediatr Dent. 2014; 15: 107-112.

17. Joshi N, Sujan S, Joshi K, Parekh H, Dave B. Prevalence, severity and related factors of dental caries in school going children of Vadodara city - an epidemiological study. J Int Oral Health. 2013; 5: 35-39.

18. Bourgeois DM, Llodra JC. Global burden of dental condition among children in nine countries participating in an international oral health promotion programme, 2012-2013. Int Dent J. 2014; 64 (Suppl 2): 27-34.

19. Kramer PF, Chaffee BW, Bertelli AE, Ferreira SH, Béria JU, Feldens CA. Gains in children's dental health differ by socioeconomic position: evidence of widening inequalities in southern Brazil. Int $\mathrm{J}$ Paediatr Dent. 2014; [E11 ahead of print].

20. Shailee F, Sogi GM, Sharma KR, Nidhi P. Dental caries prevalence and treatment needs among 12and 15- Year old schoolchildren in Shimla city, Himachal Pradesh, India. Indian J Dent Res. 2012; 23: 579-584.

21. Jeremias F, de Souza JF, Silva CM, Cordeiro Rde C, Zuanon AC, Santos-Pinto L. Dental caries experience and Molar-Incisor Hypomineralization. Acta Odontol Scand. 2013; 71: 870-876.

22. Grošelj M, Jan J. Molar incisor hypomineralisation and dental caries among children in Slovenia. Eur J Paediatr Dent. 2013; 14: 241-245.

23. Lopes RM, Domingues GG, Junqueira SR, Araujo ME, Frias AC. Conditional factors for untreated caries in 12-year-old children in the city of São Paulo. Braz Oral Res. 2013; 27: 376-381.

24. Krisdapong S, Prasertsom P, Rattanarangsima K, 
Sheiham A. Sociodemographic differences in oral health-related quality of life related to dental caries in thai school children. Community Dent Health. 2013; 30: 112-118.

25. Carta G, Cagetti MG, Sale S, Congiu G, Strohmenger $\mathrm{L}$, Oleari F, et al. Oral health inequalities in Italian schoolchildren--a cross-sectional evaluation. Community Dent Health. 2014; 31: 123-128.

26. Sgan-Cohen HD, Margvelashvili V, Bilder L, Kalandadze M, Gordon M, Margvelashvili M, et al. Dental caries among children in Georgia by age, gender, residence location and ethnic group. Community Dent Health. 2014; 31(3): 163-166.

27. Bernabé E, Sheiham A. Age, period and cohort trends in caries of permanent teeth in four developed countries. Am J Public Health. 2014; 104: e115-121.

28. Vallejos-Sánchez AA, Medina-Solís CE, MinayaSánchez M, Villalobos-Rodelo JJ, Marquez-Corona ML, Islas-Granillo H, et al. Maternal characteristics and treatment needs as predictors of dental health services utilization among Mexican school children. Eur J Paediatr Dent. 2012; 13: 307-310.

29. Vallejos-Sánchez AA, Medina-Solís CE, CasanovaRosado JF, Minaya-Sánchez M, Robles-Minaya JL, De la Rosa-Santillana R, et al. Prevalencia y distribución de los defectos de desarrollo del esmalte en dentición permanente de escolares de 6 a 12 años. Rev Latin Ortod Odont. 2012.

30. Vallejos-SánchezAA, Medina-Solís CE, Maupomé G, Casanova Rosado JF, Minaya-Sánchez M, VillalobosRodelo JJ, et al. Socio-behavioral factors influencing tooth brushing frequency in schoolchildren. J Ame Dent Assoc. 2008; 139: 743-749.

31. Vallejos-Sánchez AA, Medina-Solís CE, CasanovaRosado JF, Maupomé G, Casanova-Rosado AJ, Minaya-Sánchez M. Defectos del esmalte, caries en dentición primaria, fuentes de fluoruro y su relación con la presencia de caries en dientes permanentes. Gac Sanit. 2007; 21: 227-234.

32. Vallejos-Sánchez AA, Medina-Solís CE, CasanovaRosado JF, Maupomé G, Minaya-Sánchez M, Pérez-Olivares S. Dental fluorosis in cohorts born before, during and after the national salt fluoridation program in a community in Mexico. Acta Odontol Scand 2006; 64: 209-213.

33. Gutiérrez-Salazar M, Morales RJ. Validación de un indicador predictivo de riesgo de aparición de caries en dientes permanentes. Rev Med Distr Fed Mex. 1987; 4: 183-187.

34. FDI. An epidemiological index of development defects of dental enamel (DDE index). Int Dent J. 1982; 32: 159-167.

35. Lukacs JR, Largaespada LL. Explaining sex differences in dental caries prevalence: saliva, hormones, and "life-history" etiologies. Am J Hum Biol. 2006; 18: 540-555.

36. Lukacs JR. Gender differences in oral health in South Asia: metadata imply multifactorial biological and cultural causes. Am J Hum Biol. 2011; 23: 398-411.

37. Lukacs JR. Sex differences in dental caries experience: clinical evidence, complex etiology. Clin Oral Investig. 2011; 15: 649-656.

38. Pérez-Olivares SA, Gutiérrez-Salazar MP, SotoCantero L, Vallejos-Sánchez AA, Casanova-Rosado JF. Caries dental en primeros molares permanentes y factores socioeconómicos en escolares de Campeche, México. Rev Cubana Estomatol. 2002; 39: $265-281$.

39. Navas PR, Rojas de Morales T, Zambrano O, Álvarez CJ, Santana Y, Viera N. Salud bucal en preescolares: su relación con las actitudes y nivel educativo de los padres. Interciencia. 2002; 27: 631-634.

40. Saldūnaitė K, Bendoraitienè EA, Slabšinskienė E, Vasiliauskienė I, Andruškevičienè V, Zūbienè J. The role of parental education and socioeconomic status in dental caries prevention among Lithuanian children. Medicina (Kaunas). 2014; 50: 156-161.

41. American Academy of Pediatric Dentistry, Council on Clinical Affairs. Policy on the dental home. 2011; 36(6): 14-15.

42. American Academy of Pediatrics. Oral health risk assessment timing and establishment of the dental home. AAP Policy Statement. Pediatrics. 2003; 111: 1113-1116.

43. Pontigo-Loyola AP, Medina-Solís CE, MárquezCorona ML, Vallejos-Sánchez AA, MinayaSánchez M, Escoffié-Ramirez M, et al. Influencia de variables predisponentes, facilitadoras y de necesidades sobre la utilización de servicios de salud bucal en adolescentes mexicanos en un medio semi-rural. Gac Med Mex. 2012; 148: 218-226.

44. Medina-Solis CE, Maupomé G, Herrera MS, AvilaBurgos L, Pérez-Núñez R, Lamadrid-Figueroa $\mathrm{H}$. Dental health services utilization in children 6-12 year old in a low-income country. J Public Health Dent. 2008; 68: 39-45.

45. Jürgensen N, Petersen PE. Oral health and the impact of socio-behavioural factors in a cross sectional survey of 12-year old school children in Laos. BMC Oral Health. 2009; 9: 29.

46. Ayele FA, Taye BW, Ayele TA, Gelaye KA. Predictors of dental caries among children 7-14 years old in Northwest Ethiopia: a community based cross-sectional study. BMC Oral Health. 2013; 13: 17. 